\title{
Qualidade do sono e workaholism em docentes de pós-graduação stricto sensu
}

Sleep quality and workaholism in stricto sensu graduate professors Calidad del sueño y workaholism en docentes de posgrado stricto sensu

\author{
Mariana Guimarães Cardoso ${ }^{1}$ iD https://orcid.org/0000-0002-6447-469x \\ Arthur Eumann Mesas ${ }^{1}$ id https://orcid.org/0000-0002-0088-8607 \\ Alexandrina Aparecida Maciel Cardelli'1 io https://orcid.orgy/0000-0002-0222-8821 \\ Maria José Quina Galdino² io https://orcid.org/0000-0001-6709-3502 \\ Maynara Fernanda Carvalho Barreto ${ }^{1}$ io https://orcid.org/0000-0002-3562-8477 \\ Patricia Aroni ${ }^{1}$ is https://orcid.org/0000-0001-5092-2714 \\ Maria do Carmo Fernandez Lourenço Haddad ${ }^{1}$ io https://orid.org/0000-0001-7564-8563
}

\section{Como citar:}

Cardoso MG, Mesas AE, Cardelli AA, Galdino

MJ, Barreto MF, Aroni P. Qualidade do sono e workaholism em docentes de pósgraduação stricto sensu. Acta Paul Enferm. 2020;33:AAPE20190228.

DOI

http://dx.doi.org/10.37689/actaape/2020A002285

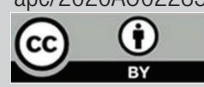

Descritores

Saúde do trabalhador; Sono; Educação de pósgraduação; Condições de trabalho; Docentes

Keywords

Occupational health; Sleep; Education, graduate Working conditions; Faculty

Descriptores

Salut laboral; Sueño; Educación de postgrado; Condiciones de trabajo; Docentes

\section{Submetido \\ 5 de Agosto de 2019 \\ Aceito \\ 19 de Fevereiro de 2020}

\section{Autor correspondente}

Maria José Quina Galdino E-mail:mjggaldino@gmail.com

\section{Resumo}

Objetivo: investigar a associação entre a qualidade do sono e 0 workaholism em docentes de pós-graduação stricto sensu.

Métodos: estudo transversal desenvolvido com docentes de todas as grandes áreas de conhecimento de uma universidade pública localizada na Região Sul do Brasil. A coleta de dados ocorreu no período de maio a agosto de 2018, por meio de formulário eletrônico contendo um questionário de caracterização sociodemográ́ica e ocupacional, a Dutch WorkAddiction Scale e o Pittsburgh Sleep Quality Index. Foram convidados os 790 docentes de pós-graduação stricto sensu da referida instituição, dos quais 196 consentiram a participação na pesquisa. Os dados foram analisados descritivamente e por regressão logística binária univariada e mútipla, adotando-se nível de significância de 95\%.

Resultados: a qualidade do sono ruim esteve associada ao trabalho excessivo $\left(0 R^{a}: 2,056 ; p=0,026\right)$ e ao workaholism $\left(0 R^{\mathrm{a}}: 2,056 ; \mathrm{p}=0,040\right)$. Qualidade subjetiva do sono, latência do sono, duração do sono, distúrbios do sono e sonolência diurna associaram-se significativamente ao trabalho excessivo e compulsivo, bem como ao workaholism.

Conclusão: as altas demandas de trabalho características do processo de trabalho dos docentes em nível stricto sensu podem repercutir na baixa qualidade do sono, visto que os docentes workaholics apresentaram duas vezes a chance de apresentarem má qualidade do sono.

\section{Abstract}

Objective: to investigate the association between sleep quality and workaholism in stricto sensu graduate professors.

Methods: a cross-sectional study developed with professors from all major areas of knowledge at a public university located in the South Region of Brazil. Data collection took place from May to August 2018, using an electronic form containing a questionnaire for sociodemographic and occupational characterization, the Dutch Work Addiction Scale and the Pittsburgh Sleep Quality Index. The 790 stricto sensu graduate professors of the institution were invited, of whom 196 consented to participate in the research. Data were analyzed descriptively and by univariate and multiple binary logistic regression, adopting a significance level of $95 \%$.

Results: poor sleep quality was associated with overwork ( $\left(\mathrm{R}^{\mathrm{Adj}}\right.$ : 2,056; $\left.\mathrm{p}=0.026\right)$ and workaholism ( $\mathrm{O} \mathrm{R}^{\mathrm{Adj}}$ : 2,056; $\mathrm{p}=0.040$ ). Subjective sleep quality, sleep latency, sleep duration, sleep disorders and daytime sleepiness were significantly associated with over and compulsive work, as well as workaholism.

Conclusion: high work demands characteristic of the professors' work process at stricto sensu level may have an impact on the low sleep quality, since workaholic professors presented twice the chance of presenting poor sleep quality.

Descriptors: Worker's health; Sleep; Professors; Graduate Education; Work conditions. 


\section{Resumen}

Objetivo: Investigar la relación entre la calidad del sueño y workaholism en docentes de posgrado stricto sensu.

Métodos: Estudio transversal que se llevó a cabo con docentes de todas las grandes áreas de conocimiento de una universidad pública ubicada en la región Sur de Brasil. La recolección de datos se realizó en el período de mayo a agosto de 2018, mediante formulario electrónico que contenía un cuestionario de caracterización sociodemográfica y ocupacional, la Dutch Work Addiction Scale y el Pittsburgh Sleep Quality Index. Se invitó a los 790 docentes de posgrado stricto sensu de la institución mencionada, de los cuales 196 aceptaron participar en la investigación. Los datos fueron analizados descriptivamente y por regresión logística binaria univariada y múltiple, con un nivel de significación de 95 \%.

Resultados: La mala calidad de sueño se relacionó con el trabajo excesivo $\left(\mathrm{OR}^{\mathrm{aj}}: 2,056 ; p=0,026\right)$ y con el workaholism $\left(0 \mathrm{R}^{\mathrm{aj}}: 2,056 ; \mathrm{p}=0,040\right)$. La calidad subjetiva del sueño, la latencia del sueño, la duración del sueño, los trastornos de sueño y la somnolencia diurna se relacionan significativamente con el trabajo excesivo y compulsivo, así como con el workaholism.

Conclusión: Las altas demandas de trabajo, características del proceso laboral de los docentes de nivel stricto sensu, pueden repercutir en la baja calidad del sueño, ya que los docentes workaholics presentan el doble de probabilidad de tener un sueño de mala calidad.

\section{Introdução}

O fenômeno workaholism foi citado pela primeira vez em 1971 por Oates e é definido como uma necessidade irresistível ou incontrolável de trabalhar incessantemente. Esse termo tem se evidenciado devido às mudanças que caracterizam a vida profissional moderna, em que os processos de trabalho estão atrelados ao uso extenuante das tecnologias da informação e comunicação (TIC). ${ }^{(1)}$

Devido às distraçóes originadas pelas TIC, seja para fins sociais, de lazer ou laborais, as horas de sono são comprometidas. Devido a vários fatores, tem-se uma perda de cerca de $25 \%$ do horário de sono quando comparado há 100 anos atrás. ${ }^{(2)}$ Este fato causa impactos negativos para o indivíduo, pois a perda da função reparadora do sono compromete a restauração das condições física e psicológica do organismo, desgastadas durante o estado de vigília. ${ }^{(3)}$

O trabalho docente é influenciado pelas transformaçóes contemporâneas e a educação superior tornou-se uma alavanca para o desenvolvimento econômico do país e o conhecimento passou a ser visto por meio da produção científica desses trabalhadores. ${ }^{(4,5)}$ Nesse contexto, o processo de trabalho docente passou a demandar profissionais imediatistas, resolutivos e atentos a atualizaçôes constantes. $\mathrm{O}$ meio científico passa a existir numa temporalidade de aceleraçáo permanente em busca de produtividade e competência, em que se acentuam os dispositivos de controle que enfocam ligaçóes funcionais e pragmáticas do processo de trabalho do professor em nível stricto sensu, tais como sistemas de avaliação e plataformas integradas de currículo. ${ }^{(6)}$

Todo este cenário propicia uma jornada de trabalho que se estende além do ambiente profissional, aumentando de forma extrema o risco de trabalho excessivo e investimento laboral insalubre, ${ }^{(7)}$ aspectos característicos do workaholism, compreendido neste estudo pelas dimensóes de trabalho excessivo e compulsivo. ${ }^{(8)}$

Acredita-se que o workaholism afete a qualidade do sono, pois o ritmo de trabalho é um dos fatores que mais afeta a arquitetura dos estágios de sono. ${ }^{(9)}$ Nesse sentido, as longas jornadas laborais e a carga horária semanal elevada associam-se a prejuízos a qualidade do sono. ${ }^{(10,11)}$

O sono é um estado biocomportamental naturalmente recorrente e reversível, caracterizado por imobilidade relativa, desengajamento perceptivo e consciência moderada, ${ }^{(9)}$ que é um dos principais responsáveis pela restauração da energia e dos recursos mentais do ser humano. Desse modo, dormir bem possibilita às pessoas capacidade e prontidão para atender as demandas de trabalho atuais e novas. Por outro lado, entre professores, a ausência de recuperaçáo pelo sono e lazer aumentam o estresse laboral e a exaustão. ${ }^{(12)}$

Mediante a relevância desta temática para o bem-estar do professor e tendo em vista a escassez de publicaçóes no contexto do fenômeno workaholism no impacto na saúde do trabalhador, principalmente dos docentes de pós-graduação stricto sensu, espera-se que os dados apresentados neste estudo embasem futuras pesquisas sobre a qualidade de vida física e mental dos indivíduos por trás 
da difusão do conhecimento da ciência, pensando não só na produtividade científica, mas na saúde do profissional.

Considerando os aspectos do fenômeno workaholism apresentados e as características laborais do trabalho docente, esse estudo objetiva investigar a associação entre o workaholism e a qualidade do sono em docentes de pós-graduação stricto sensu.

\section{Métodos}

Trata-se de um estudo transversal desenvolvido em uma universidade pública localizada na Região Sul do Brasil. A referida instituição de ensino conta com 52 cursos presenciais de Graduação (bacharelados e licenciaturas) e 261 cursos de Pós-Graduação (PPG), dos quais 186 são lato sensu (residências e especializaçóes) e 75 em nível stricto sensu (mestrados e doutorados), distribuídos em nove centros de estudos, abrangendo diversas áreas de conhecimento.

A população deste estudo constituiu-se de todos os 790 docentes vinculados à pelo menos um dos programas de pós-graduação stricto sensu da referida instituição. Os critérios de elegibilidade foram estar vinculado há um programa de pós-graduação stricto sensu da universidade em estudo e não estar afastado das atividades por licenças laborais.

A coleta de dados ocorreu no período de 16 de maio a 30 de agosto de 2018 por meio de formulário eletrônico. $\mathrm{O}$ contato com os docentes foi estabelecido por meio do e-mail cadastrado no site institucional do PPG. Foram realizados convites a todos os 790 docentes a cada 15 dias, sendo limitados à cinco envios, respondendo ao questionário 196 (24,8\%), os quais atendiam aos critérios de elegibilidade do estudo.

As variáveis sociodemográficas (sexo, idade e estado civil), de hábitos de vida (atividade física, tabagismo e oportunidades de lazer) e ocupacionais (formação, vínculo e regime de trabalho, anos de atividade no ensino superior e na pós-graduação, número de vínculos em PPG, conceito Coordenação de Aperfeiçoamento de Pessoal de Nível Superior - CAPES do PPG, bolsista produtividade, horas destinadas à pós-graduação, atividades de trabalho além da carga horária, número de orientandos de mestrado/doutorado, número de artigos publicados ou aceitos no último ano, relacionamento com os professores e orientandos do PPG, ritmo de intensidade em relação ao trabalho, se já pensou em deixar o PPG, preocupaçóes e/ou dificuldades em relação ao trabalho) foram mensuradas por meio de um questionário semiestruturado, elaborado pelos autores.

Para avaliar o workaholism foi utilizada a versão reduzida da Dutch Work Addiction Scale (DUWAS), elaborada por Schaufeli, Taris and Bakker ${ }^{(8)}$ e validada no Brasil por Carlotto e Del Líbano ${ }^{(13)}$ em profissionais brasileiros. Trata-se de um instrumento constituído por 10 itens avaliados com uma escala tipo Likert, variando de 1 (nunca) a 4 (sempre). A escala avalia duas dimensôes independentes que não produzem uma pontuação composta: trabalho compulsivo (itens 1, 2, 4, 6 e 8) e trabalho excessivo (itens 3, 5, 7, 9 e 10), no entanto a presença simultânea de alto trabalho compulsivo e excessivo indicam workaholism. Pela ausência de estudos com docentes brasileiros, a confiabilidade da DUWAS foi calculada neste estudo pelo coeficiente alfa de Cronbach, sendo que o trabalho compulsivo obteve $\alpha=0,795$ e trabalho excessivo $\alpha=0,782$.

A qualidade do sono foi verificada pelo Pittsburgh Sleep Quality Index (PSQI), um instrumento elaborado por Buysse e colaboradores, ${ }^{(14)}$ traduzido e validado para o Brasil por Bertolazi. ${ }^{(15)} \mathrm{O}$ instrumento mede a percepção da qualidade do sono referente ao mês anterior por meio de 19 questóes divididas em sete componentes: qualidade subjetiva do sono (item 6), latência do sono (itens 2 e 5a), duração do sono (item 4); eficiência habitual do sono (itens 1, 3 e 4); distúrbios do sono (itens $5 \mathrm{~b}$ até $5 \mathrm{j}$ ); uso de medicaçóes para dormir (item 7) e sonolência diurna (itens 8 e 9). As respostas são fornecidas em escala do tipo Likert quatro pontos (0-3). A soma dos componentes origina um escore total que varia de 0 a 21 pontos. Considerando que não encontrou-se estudos com professores de nível superior, calculou-se a confiabilidade do PSQI neste estudo pelo coeficiente alfa de Cronbach, $\alpha=0,774$.

Os dados foram analisados no Statistical Package of Social Sciences (SPSS), versão 20.0 por estatísti- 
ca descritiva e inferencial. As variáveis dependentes deste estudo foram o escore geral da qualidade do sono, que foi dicotomizado em ruim ( $>5$ pontos) e bom ( $\leq 5$ pontos), conforme o referencial teórico-metodológico adotado; ${ }^{(14)}$ e os componentes do PSQI. As variáveis independentes foram as dimensões da DUWAS, trabalho excessivo e compulsivo, dicotomizadas considerando a mediana como ponto de corte. Altos níveis em ambas dimensôes indicaram workaholism. ${ }^{(16)}$

A associação das variáveis dependentes com as independentes foram verificadas por regressão logística univariada. Posteriormente, por regressão logística múltipla, essa associação foi ajustada por sexo e idade ${ }^{(17,18)}$ e por aspectos relativos ao processo de trabalho docente que ajustavam os valores de $\beta 1$ em, no mínimo, $10 \%$. O odds ratio com intervalo de confiança de $95 \%$ foi selecionado como medida de associação e considerou-se $\alpha<0,05$ como estatisticamente significativo.

O estudo seguiu a Resolução $n^{\circ} 466 / 2012$ do Conselho Nacional de Saúde, sendo submetido e aprovado pelo Comitê de Ética em Pesquisa (CEP) sob Parecer $n^{\circ}$ 2.347.839. Antes de acessarem o questionário de coleta de dados, todos os docentes consentiram a participação pelo Termo de Consentimento Livre e Esclarecido (TCLE), cujo acesso para leitura era online.

\section{Resultados}

A amostra deste estudo constituiu-se de 196 docentes de pós-graduação stricto sensu, 24,8\% da população alvo. A maioria era do sexo feminino $(60,7 \%)$, casados (76\%), cuja idade variou entre 32 e 75 anos. Com relação aos hábitos de vida, 37,2\% foram considerados ativos fisicamente, $7,7 \%$ eram tabagistas e $88,8 \%$ referiram poucas oportunidades de lazer.

Quanto à formação, segundo as áreas de conhecimento do Conselho Nacional de Desenvolvimento Científico e Tecnológico (CNPq), 33,7\% (66) eram das Ciências da Saúde, 11,7\% (23) Ciências Humanas, 11,2\% (22) Ciências Biológicas, 10,7\% (21) Ciências Sociais Aplicadas, 10,2\% (21) Ciências Exatas e da Terra, 9,2\% (18) Ciências
Agrárias, 7,7\% (15) Linguística, Letras e Artes e 5,6\% (11) Engenharias. Sobre o vínculo e o regime de trabalho na universidade, 89,3\% (175) possuíam dedicação exclusiva em tempo integral, $4,6 \%$ (9) tempo integral, 4,1\% (8) tempo parcial e 2,0\% (4) atuavam como professor sênior. A média de anos de docência no ensino superior foi de 19 anos $( \pm 9,1)$, variando entre 02 e 45 anos.

Entre as variáveis sociodemográficas, hábitos de vida e do processo de trabalho docente apenas a influência negativa do ritmo e intensidade do trabalho $(\mathrm{p}=0,010)$ foi associada à qualidade do sono ruim (Tabela 1 ).

A prevalência de qualidade do sono ruim foi de $65,8 \%$ (129), alto trabalho excessivo de 54,1\% (106), alto trabalho compulsivo de 51,5\% (101) e workaholism de 13,1\% (26). A qualidade do sono ruim foi associada ao alto trabalho excessivo e ao workaholism, mesmo após os ajustes (Tabela 2).

$\mathrm{Na}$ tabela 3, verificou-se que o trabalho excessivo, compulsivo e ao workaholism contribuíram para a redução da qualidade subjetiva do sono, latência do sono, duração do sono e aumento dos distúrbios do sono e sonolência diurna.

Ressalta-se que 21,9\% dos docentes referiram utilizar alguma medicação para dormir (prescrita ou não por médico). Os principais medicamentos citados pelos participantes pertenciam as classes de ansiolíticos, relaxantes musculares, hipnóticos, fitoterápicos/homeopáticos, analgésicos, antidepressivos e o hormônio melatonina.

\section{Discussão}

A qualidade de sono ruim esteve associada ao trabalho excessivo e ao workaholism, mesmo após os ajustes número de PPG que atua, nível do(s) curso(s) de vínculo do docente, pressão pelo bom desempenho profissional, pressão por publicação científica, ser bolsista produtividade, sexo e idade. Infere-se que o processo de trabalho docente caracterizado pelas variáveis de ajuste mencionadas e as exigências de produtividade podem induzir os docentes a desenvolver muitos projetos concomitantes ou acelerar o ritmo de trabalho. ${ }^{(20)}$ Além disso, tendo em vista todo este 
Tabela 1. Associação entre qualidade do sono e variáveis sociodemográficas, hábitos de vida e do processo de trabalho de docentes de pós-graduação stricto sensu

\begin{tabular}{|c|c|c|c|c|}
\hline \multirow{2}{*}{ Variáveis } & \multicolumn{2}{|c|}{ Qualidade do sono } & \multirow{2}{*}{$\mathrm{p}$-value } & \multirow{2}{*}{$\begin{array}{l}\text { Odds ratio (intervalo de } \\
\text { confiança de } 95 \% \text { ) }\end{array}$} \\
\hline & Ruim & Boa & & \\
\hline \multicolumn{5}{|l|}{ Sexo } \\
\hline Masculino & $52(67,5)$ & $25(32,5)$ & 0,684 & 1 \\
\hline Feminino & $77(64,7)$ & $42(35,3)$ & & $0,881(0,480-1,618)$ \\
\hline Idade (em anos) & $48 \pm 9,1$ & & 0,624 & $0,992(0,960-1,025)$ \\
\hline \multicolumn{5}{|l|}{ Estado Civil } \\
\hline Solteiros/separados/viúvos & $30(63,8)$ & $17(36,2)$ & 0,742 & 1 \\
\hline Casados & $99(66,4)$ & $50(33,6)$ & & $1,122(0,565-2,226)$ \\
\hline \multicolumn{5}{|l|}{ Ativo Fisicamente* } \\
\hline Não & $81(65,9)$ & $42(34,1)$ & 0,989 & 1 \\
\hline $\operatorname{Sim}$ & $48(65,8)$ & $25(34,2)$ & & $0,996(0,541-1,833)$ \\
\hline \multicolumn{5}{|l|}{ Tabagismo } \\
\hline Não & $118(65,2)$ & $63(34,8)$ & 0,525 & 1 \\
\hline Sim & $11(73,3)$ & $4(26,7)$ & & $1,468(0,449-4,800)$ \\
\hline \multicolumn{5}{|l|}{ Lazer } \\
\hline Muitas oportunidades & $12(54,5)$ & $10(45,5)$ & 0,241 & 1 \\
\hline Poucas oportunidades & $117(67,2)$ & $57(32,8)$ & & $1,711(0,698-4,194)$ \\
\hline \multicolumn{5}{|l|}{ Pós-doutorado } \\
\hline Não & $79(66,4)$ & $40(33,6)$ & 0,834 & 1 \\
\hline $\operatorname{Sim}$ & $50(64,9)$ & $27(35,1)$ & & $0,938(0,513-1,714)$ \\
\hline Anos de docência no nível stricto sensu & $9,4 \pm 7,1$ & & 0,490 & $0,986(0,946-1,027)$ \\
\hline Número de PPG vinculados & $1,3 \pm 0,5$ & & 0,051 & $0,591(0,348-1,003)$ \\
\hline \multicolumn{5}{|l|}{ Conceito Capes do PPG } \\
\hline $3-4$ & $93(64,6)$ & $51(35,4)$ & 0,545 & 1 \\
\hline $5-7$ & $36(69,2)$ & $16(30,8)$ & & $1,234(0,625-2,437)$ \\
\hline \multicolumn{5}{|l|}{ Curso(s) de vínculo do docente } \\
\hline Mestrado & $42(75,0)$ & $14(25,0)$ & 0,089 & 1 \\
\hline Mestrado e doutorado & $87(62,1)$ & $53(37,9)$ & & $0,547(0,273-1,096)$ \\
\hline \multicolumn{5}{|l|}{ Bolsista produtividade } \\
\hline Não & $102(65,8)$ & $53(34,2)$ & 0,995 & 1 \\
\hline Sim & $27(65,9)$ & $14(34,1)$ & & $1,002(0,485-2,071)$ \\
\hline Horas dedicadas à pós-graduação stricto sensu & $13,6 \pm 10,2$ & & 0,433 & $0,989(0,961-1,017)$ \\
\hline Horas dedicadas ao trabalho fora do contratado & $14,4 \pm 10,5$ & & 0,100 & $1,027(0,995-1,060)$ \\
\hline Número de orientados no nível stricto sensu & $4,6 \pm 2,8$ & & 0,734 & $0,982(0,885-1,090)$ \\
\hline Número de artigos publicados ou aceitos no último ano & $4,8 \pm 4,5$ & & 0,064 & $0,940(0,880-1,004)$ \\
\hline \multicolumn{5}{|l|}{ Relacionamento interpessoal com os professores do PPG } \\
\hline Excelente/bom & $112(64,4)$ & $62(35,6)$ & 0,235 & 1 \\
\hline Regular/ruim & $17(77,3)$ & $5(22,7)$ & & $1,882(0,662-5,348)$ \\
\hline \multicolumn{5}{|l|}{ Relacionamento interpessoal com seus orientandos } \\
\hline Excelente/bom & $125(66,1)$ & $64(33,9)$ & 0,624 & 1 \\
\hline Regular/ruim & $4(57,1)$ & $3(42,9)$ & & $0,683(0,148-3,143)$ \\
\hline \multicolumn{5}{|l|}{ Ritmo e intensidade do trabalho } \\
\hline Influência positiva & $50(56,2)$ & $39(43,8)$ & 0,010 & 1 \\
\hline Influência negativa & $79(73,8)$ & $28(26,2)$ & & $2,201(1,207-4,014)$ \\
\hline \multicolumn{5}{|l|}{ Intenção de deixar a pós-graduação stricto sensu } \\
\hline Não & $44(58,7)$ & $31(41,3)$ & 0,098 & 1 \\
\hline Sim & $85(70,2)$ & $36(29,8)$ & & $1,664(0,911-3,039)$ \\
\hline \multicolumn{5}{|l|}{ Pressão para publicação científica } \\
\hline Não & $75(62,5)$ & $45(37,5)$ & 0,416 & 1 \\
\hline Sim & $54(71,1)$ & $22(28,9)$ & & $1,300(0,691-2,446)$ \\
\hline
\end{tabular}

Fonte: Lima DF, Levy RB, Luiz OC. Recommendations for physical activity and health: consensus, controversies, and ambiguities. Rev Panam Salud Publica. 2014;36(3):164-70.(19)

*a classificação seguiu as recomendações da Organização Mundial de Saúde, sendo considerados ativos, os que realizaram $\geq 150$ minutos de atividades físicas leves/moderadas/intensas, no mínimo duas vezes por semana. ${ }^{19}$

Tabela 2. Associação da qualidade do sono com o workaholism em docentes da pós-graduação stricto sensu

\begin{tabular}{lcccc}
\hline Variáveis & p-value & $\begin{array}{c}\text { Odds ratiobrto } \\
\text { (intervalo de confiança 95\%) }\end{array}$ & p-value & $\begin{array}{c}\text { Odds ratioaiustado* } \\
\text { (intervalo de confiança 95\%) }\end{array}$ \\
\hline Qualidade do sono & & & & \\
$\quad$ Trabalho excessivo & 0,009 & $2,280(1,232-4,222)$ & 0,026 & $2,056(1,092-3,874)$ \\
Trabalho compulsivo & 0,052 & $1,813(0,994-3,307)$ & 0,053 & $1,844(0,991-3,431)$ \\
Workaholism & 0,022 & $2,194(1,117-4,309)$ & 0,040 & $2,056(1,032-4,098)$ \\
\hline
\end{tabular}

"ajustado por número de Programas de Pós-Graduação, nível do(s) curso(s) de vínculo do docente, pressão pelo bom desempenho profissional, pressão para publicação científica, bolsista produtividade, uso de antidepressivos, sexo e idade 
Tabela 3. Associação dos componentes da qualidade do sono com 0 workaholism em docentes da pós-graduação stricto sensu

\begin{tabular}{|c|c|c|}
\hline Variáveis & $p$-value & $\begin{array}{l}\text { Odds ratiobruto } \\
\text { (intervalo de confiança 95\%) }\end{array}$ \\
\hline \multicolumn{3}{|c|}{ Qualidade subjetiva do sono } \\
\hline Trabalho excessivo & $<0,001$ & $3,609(1,942-6,706)$ \\
\hline Trabalho compulsivo & $<0,001$ & $3,371(1,812-6,270)$ \\
\hline Workaholism & $<0,001$ & $3,465(1,850-6,487)$ \\
\hline \multicolumn{3}{|l|}{ Latência do sono } \\
\hline Trabalho excessivo & 0,009 & $2,229(1,224-4,058)$ \\
\hline Trabalho compulsivo & 0,017 & $2,281(1,143-3,789)$ \\
\hline Workaholism & 0,041 & $1,900(1,926-3,518)$ \\
\hline \multicolumn{3}{|l|}{ Duração do sono } \\
\hline Trabalho excessivo & 0,011 & $2,689(1,251-5,779)$ \\
\hline Trabalho compulsivo & $<0,001$ & $4,616(1,975-10,785)$ \\
\hline Workaholism & $<0,001$ & $3,989(1,866-8,528)$ \\
\hline \multicolumn{3}{|l|}{ Eficiência habitual do sono } \\
\hline Trabalho excessivo & 0,193 & $1,820(0,739-4,480)$ \\
\hline Trabalho compulsivo & 0,136 & $2,009(0,802-5,033)$ \\
\hline Workaholism & 0,198 & $1,803(0,735-4,426)$ \\
\hline \multicolumn{3}{|l|}{ Distúrbios do sono } \\
\hline Trabalho excessivo & $<0,001$ & $3,655(1,875-7,124)$ \\
\hline Trabalho compulsivo & 0,002 & $2,814(1,459-5,427)$ \\
\hline Workaholism & 0,002 & $2,737(1,429-5,243)$ \\
\hline \multicolumn{3}{|l|}{ Sonolência diurna } \\
\hline Trabalho excessivo & $<0,001$ & $3,626(1,785-7,366)$ \\
\hline Trabalho compulsivo & 0,025 & $2,171(1,100-4,285)$ \\
\hline Workaholism & 0,017 & $2,281(1,158-4,494)$ \\
\hline \multicolumn{3}{|c|}{ Uso de medicação para dormir } \\
\hline Trabalho excessivo & 0,143 & $1,665(0,842-3,292)$ \\
\hline Trabalho compulsivo & 0,457 & $1,294(0,657-2,549)$ \\
\hline Workaholism & 0,316 & $1,439(0,710-2,881)$ \\
\hline
\end{tabular}

cenário de produtividade e aumento da competitividade na área acadêmica os trabalhadores com maior demanda podem ser vistos como modelo. ${ }^{(21)}$

Com relação aos componentes da qualidade do sono, a qualidade subjetiva do sono, latência do sono, duração do sono, distúrbios do sono e sonolência diurna estiveram associados ao trabalho excessivo, compulsivo e ao workaholism. O indivíduo workaholic é compelido a esgotar seus recursos em atividades laborais. Como o sono desempenha a função biológica responsável pela ação restauradora do cansaço físico e mental sua ausência pode aumentar a atividade do sistema nervoso simpático. ${ }^{(6,22)}$

Apesar de estudos relatarem distúrbios do sono como uma possível decorrência do workaholism deve-se considerar que ambos constructos foram mensurados por instrumentos distintos, inviabilizando comparaçóes. ${ }^{(6,23)}$ Contudo, é possível afirmar que os distúrbios do sono têm potenciais efeitos na capacidade para a saúde laboral, conforme demonstrou pesquisa norueguesa, na qual, indivíduos que trabalham em horários não convencionais, ou seja, possuem um ritmo circadiano flexível eram mais propensos a desenvolver workaholism. ${ }^{(24)}$

Estudo realizado no Estado do Rio Grande do Norte constatou que um em cada três professores universitários foram identificados com sonolência diurna excessiva. Infere-se que isto possa ser reflexo de um ritmo de vida acelerado a que esses profissionais estão submetidos, em que devem preparar e ministrar aulas, preparar, aplicar e corrigir provas, produzir artigos, elaborar trabalhos de pesquisas, orientar alunos, entre outras atividades. Essa alta demanda de trabalho ainda pode estar associada à latência do sono (período de tempo necessário para realizar a transição da vigília para o sono total), pois o trabalho realizado até altas horas impossibilita uma higiene adequada do sono, interferindo no tempo destinado ao repouso desse docente, levando-o ao hábito de se privar de horas de sono que seriam essenciais. ${ }^{(25)}$

Esses hábitos podem ter consequências a curto e a longo prazo na saúde e segurança dos trabalhadores, pois ocasionam queda das funçôes cognitivas, no raciocínio e no julgamento, tornando-os mais propensos a erros, ${ }^{(26)}$ e prejudicando o processo de trabalho docente que envolve majoritariamente aspectos cognitivos.

Pesquisas indicam que a dimensão compulsiva desencadeia sentimento de ansiedade que pode interferir na duração do sono. Ressaltam-se como consequências negativas a exaustão física e emocional, que são as principais precursoras do estresse e da síndrome de burnout e, por consequência, deterioram a saúde mental do trabalhador. ${ }^{(22,27)}$

A eficiência do sono e o uso da medicação para dormir não tiveram associação com as dimensóes excessiva e compulsiva do trabalho e nem com o workaholism. Todavia, os docentes referiram a utilização de medicação para dormir e sabe-se que existem algumas medidas não farmacológicas para otimizar a qualidade do sono que poderiam ser adotadas para os docentes, tais como: técnicas de relaxamento, massagem, musicoterapia, assim como orientaçóes educativas das instituições quanto a higiene do sono antes de dormir. ${ }^{(28,29)}$

Como limitação deste estudo indica-se a metodologia transversal, em que desfecho e exposição são men- 
surados simultaneamente. Ainda, o questionário de preenchimento autorreferido, cujas respostas podem ser influenciadas pelo interesse dos participantes pode ser considerado outra limitação. Todavia, o presente estudo contribui com a discussão da temática no que tange à influência do workaholism na qualidade do sono, avançando em apresentar a associação da qualidade do sono, sobretudo na avaliação dos seus componentes em relação à cada dimensão do workaholism. Para estudos futuros sugerem-se métodos longitudinais com a mensuração de dados clínicos objetivos, a fim de preencher as lacunas existentes quanto à relação em longo prazo entre workaholism e a qualidade do sono.

Como implicaçóes práticas, dadas as oportunidades limitadas de influenciar aspectos pessoais dos trabalhadores, uma alternativa aos gestores é estimular um ambiente de trabalho em que sejam adotadas políticas e práticas de pessoal que limitem os docentes a trabalhar excessivamente em nível estrutural ou fornecer recursos para equilibrar as demandas de trabalho. Ainda é importante que as agências governamentais foquem as avaliaçóes de desempenho dos PPG no seu papel social, isto é, na formação de profissionais qualificados para o avanço da ciência e da tecnologia e não na produtividade acadêmica.

\section{Conclusão}

As altas demandas de trabalho características do processo de trabalho dos docentes de pós-graduação stricto sensu podem estar associadas a qualidade do sono ruim, pois foi constatado que os docentes workaholics e com alto trabalho excessivo apresentaram chances aumentadas de má qualidade do sono, mesmo após ajustes. Dos sete componentes da qualidade do sono, qualidade subjetiva do sono, latência do sono, duração do sono, distúrbios do sono e sonolência diurna tiveram associação com o workaholism, trabalho excessivo e compulsivo.

\section{Colaborações}

Cardoso MG, Galdino MJQ e Haddad MCFL contribuíram com a concepção do projeto, análise e interpretação dos dados, redação do artigo, revisão crítica do conteúdo intelectual e aprovação final da versão a ser publicada. Mesas AE, Cardelli AAM, Barreto MFC e Aroni P contribuíram na interpretação dos dados, revisão crítica relevante do conteúdo intelectual e aprovação final da versão a ser publicada.

\section{Referências}

1. Clark MA, Stevens GS, Michel J, Zimmerman L. Workaholism among leaders: implications for their own and their followers' well-being. Role Leadersh Occup Stress. 2016;14:1-31.

2. Rodrigues M, Nina S, Matos L. Como dormimos? - Avaliação da qualidade do sono em cuidados de saúde primários. Rev Port Clin Geral. 2014;30(1):16-22.

3. Pinto AA, Felden EP, Claumann GS, Barbosa RM, Nahas MV, Pelegrini A. Sleep quality of working and physically inactive adolescents. Cad Bras Ter Ocup. 2018;26(1):137-43.

4. Maués OC, Souza MB. The transnationalization and the expansion of higher education. Rev Educ Quest. 2018;56(47):151-73.

5. Figueredo WN, Laitano AC, Santos VP, Dias AC, Silva GT, Teixeira GA. Didactic-pedagogical training in stricto sensu graduate programs in Health Sciences of Federal Universities in the Northeastern region of Brazil. Acta Paul Enferm. 2017;30(5):497-503.

6. Leite AF, Nogueira JA. Health conditioning factors work-related to health professors: an integrative review. Rev Bras Saúde Ocup. 2017;42:e6.

7. Spagnoli P, Balducci C, Scafuri Kovalchuk L, Maiorano F, Buono C. Are engaged workaholics protected against job-related negative affect and anxiety before sleep? a study of the moderating role of gender. Int J Environ Res Public Health. 2018 Sep;15(9):1996.

8. Schaufeli WB, Taris TW, Bakker AB. Dr. Jekyll or Mr. Hyde? On the differences between work engagement and workaholism. In: Burke RJ, editor. Research companion to working time and work addiction. Northampton, UK: Edward Elgar; 2006. p. 193-217.

9. Tubbs AS, Dollish HK, Fernandez F, Grandner A. The basics of sleep physiology and behavior. In: Grandner A, editor. Sleep and Health. London: Academic Press - Elsevier; 2019. p. 3-10.

10. Bannai A, Ukawa S, Tamakoshi A. Long working hours and sleep problems among public junior high school teachers in Japan. J Occup Health. 2015;57(5):457-64.

11. Hori D, Sasahara S, Oi Y, Doki S, Andrea CS, Takahashi T, et al. Relationships between insomnia, long working hours, and long commuting time among public school teachers in Japan: a nationwide cross-sectional diary study. Sleep Med. Forthcoming 2019. https://doi. org/10.1016/j.sleep.2019.09.017.

12. Gluschkoff K, Elovainio M, Kinnunen U, Mullola S, Hintsanen M, Keltikangas-Järvinen $\mathrm{L}$, et al. Work stress, poor recovery and burnout in teachers. Occup Med (Lond). 2016;66(7):564-70.

13. Carlotto MS, Miralles MD. Translation, adaptation and exploration of psychometric properties of "Dutch Work Addiction Scale" (DUWAS). Contextos Clín. 2010;3(2):141-50. 
14. Buysse DJ, Reynolds CF 3rd, Monk TH, Berman SR, Kupfer DJ. The Pittsburgh Sleep Quality Index: a new instrument for psychiatric practice and research. Psychiatry Res. 1989;28(2):193-213.

15. Bertolazi AN, Fagondes SC, Hoff LS, Dartora EG, Miozzo IC, de Barba $\mathrm{ME}$, et al. Validation of the Brazilian Portuguese version of the Pittsburgh Sleep Quality Index. Sleep Med. 2011;12(1):70-5.

16. Schaufeli WS, Shimazu A, Taris TW. Being driven to work excessively hard: the evaluation of a two-factor measure of workaholism in the Netherlands and Japan. Cross-Cultural Res. 2009;43(4):320-48.

17. Clark MA, Michel JS, Zhdanova L, Pui SY, Baltes BB. All work and no play? A meta-analytic examination of the correlates and outcomes of workaholism. J Manage. 2016;42(7):1836-73.

18. Beiler-May A, Williamson RL, Clark MA, Carter NT. Gender bias in the measurement of workaholism. J Pers Assess. 2017;99(1):104-10.

19. de Lima DF, Levy RB, Luiz OC. [Recommendations for physical activity and health: consensus, controversies, and ambiguities]. Rev Panam Salud Publica. 2014;36(3):164-70. Portuguese.

20. Souza KR, Mendonça AL, Rodrigues AM, Felix EG, Teixeira LR, Santos $\mathrm{MB}$, et al. [The new organization of labor at public universities: collective consequences of job instability on the health of teachers]. Cien Saude Colet. 2017;22(11):3667-76. Portuguese.

21. Kravina L, Falco A, De Carlo NA, Andreassen CS, Pallesen S. Workaholism and work engagement in the family: the relationship between parents and children as a risk factor. Eur J Work Organ Psychol. 2014;23(6):875-83.
22. Salanova M, López-González AA, Llorens S, Del Líbano M, VicenteHerrero MT, Tomás-Salvá M. Your work may be killing you! Workaholism, sleep problems and cardiovascular risk. Work Stress. 2016;30(3):22842.

23. Andreassen CS, Pallesen S, Torsheim T. Workaholism as a mediator between work-related stressors and health outcomes. Int J Environ Res Public Health. 2018;15(1):E73.

24. Andreassen CS, Bakker AB, Bjorvatn B, Moen BE, Magerøy N, Shimazu $A$, et al. Working conditions and individual differences are weakly associated with workaholism: a 2-3-year prospective study of shiftworking nurses. Front Psychol. 2017;8:2045.

25. Amaro JM, Dumith SC. [Excessive daytime sleepiness and quality of life related to the health of university professors]. J Bras Psiquiatr. 2018;67(2):94-100. Portuguese.

26. Balducci C, Avanzi L, Fraccaroli F. The individual "costs" of workaholism: an analysis based on multisource and prospective data. J Manage. 2016;20:1-26.

27. Sussman S. Workaholism: a review. J Addict Res Ther. 2012;6(1 Suppl 6):4120.

28. Corrêa CC, Campos LD, Weber SA. Conscientization of the habits related to sleep inside the state of São Paulo. Arch Health Invest. 2018;7(3):115-7.

29. Machado FS, Souza RC, Poveda VB, Costa AL. Non-pharmacological interventions to promote the sleep of patients after cardiac surgery: a systematic review. Rev Lat Am Enfermagem. 2017;25(25):e2926. 